\title{
Anfibios y reptiles de un humedal periurbano: El Estero, San Ramón, Costa Rica
}

\author{
Amphibians \& reptiles in a peri-urban wetland: El Estero, San Ramón, Costa Rica.
}

\author{
Melvin Cartín Núñez
}

Fecha de recepción: 21-01-2020

Fecha de aceptación: 10-09-2020

\begin{abstract}
Resumen
Con el avance de la frontera agrícola, el crecimiento urbano y el cambio climático, muchas especies de anfibios y reptiles han visto reducidas sus poblaciones. Pocos trabajos se han hecho en el medio urbano con este grupo, en particular en Costa Rica, por lo que se realizó un primer esfuerzo por generar información de línea base en la periferia de la ciudad de San Ramón, Alajuela. Se eligió la microcuenca alta de la quebrada El Estero, ubicada al oeste de la ciudad. Allí se aplicaron varios métodos de muestreo como transectos y cuadrantes de hojarasca. Además, se registraron las especies vistas en encuentros ocasionales. Luego de un esfuerzo de 120 horas persona, se contabilizaron 19 especies representantes de 14 familias, 8 de reptiles y 6 de anfibios. La mayoría de las especies fueron registradas durante los muestreos ( $63 \%, \mathrm{n}=12)$; solamente una especie, el sapo Incilius coccifer, fue registrada tanto en los muestreos como en encuentros ocasionales. Seis de las especies están asociadas a cuerpos de agua y se conocen por tolerar cierto grado de contaminación, de ahí que no sorprende su presencia en el área de estudio. El Bosque Demostrativo de la Sede de Occidente, fue el sitio con la mayor riqueza de especies registradas, probablemente por ser el sitio con mayor variedad de microhábitats para la fauna del lugar.
\end{abstract}

Palabras clave: herpetofauna, humedales, biodiversidad, riqueza de especies, medio urbano.

\begin{abstract}
Many species of amphibians and reptiles have reduced their populatios due expansion of the agricultural border, urban growth and climate change. Particularly in Costa Rica, few researchs have done in the urban environment with this group of animals. Then, this is a first effort to generate baseline data in the periphery of San Ramón city, Alajuela; at micro-basin of El Estero. Different sampling methods, as transects and leaf litter quadrants, were used. Also, species seen at occasional encounters were registered. A total of 19 species in 14 families, 8 of reptiles and 6 of amphibians, were found after 120 hours/person effort. Most of them were recorded during the samplings ( $63 \%, \mathrm{n}=12)$; only one species, Incilius coccifer toad, was registered in both, the samplings and occasional encounters. Six species are associated with water bodies and they are known to tolerate moderate contamination, so its permanence in the area is not surprising. Bosque Demostrativo at Sede de Occidente was the site of greatest species richness, because it is the place with the greatest microhabitats variety for the herpetofauna of the area.
\end{abstract}

Key word: herpetofauna, wetlands, biodiversity, species richness, urban environment.

\footnotetext{
1 Magíster en Desarrollo Sostenible, Docente de la Sección de Biología, Universidad de Costa Rica, Sede de Occidente, San Ramón, Costa Rica. Correo electrónico: melvin.cartin@ucr.ac.cr, Orcid: oooo-0oo2-2724-7498
} 


\section{Introducción}

El término herpetofauna comprende a los anfibios y reptiles vivientes, con excepción de las aves (Savage, 2002). En Costa Rica, más de 400 especies comprenden la riqueza de este grupo (436 según Bolaños et al., 2011) y por ello el país es reconocido a nivel internacional (Savage, 2002; Scott y Limerick, 1983). Sin embargo, con el avance de la frontera agrícola, el crecimiento urbano y los cambios en los patrones climáticos, muchas de las especies han visto reducidas sus poblaciones al tiempo que otras parecen persistir aún en estas condiciones desfavorables.

Si bien las ciudades costarricenses no son tan densas como las de otros países (Piedra et al., 2017), el desarrollo urbano es un fenómeno continuo y en aceleración, cuyo impacto en los ecosistemas naturales, aunque predecible en muchos casos, ha sido poco estudiado. Menos aún en grupos específicos como los anfibios y los reptiles, cuyas especies no gozan del atractivo que generan otros grupos como las aves o los mamíferos. Incluso para quienes sí simpatizan con los anfibios y reptiles, el medio urbano no goza de un especial interés para el desarrollo de investigaciones, pues generalmente se prefiere o se priorizan los espacios naturales (Gosá y Arias, 2009).

A pesar de lo anterior, conocer la riqueza de especies en áreas urbanas o periurbanas constituye la base para generar estrategias de manejo y conservación apropiadas, tal y como sucede con las áreas silvestres (DomínguezVega y Zuria, 2016). En la periferia de la ciudad de San Ramón, Costa Rica, no existen estudios previos que permitan conocer el estado de la herpetofauna local. Por ello, este trabajo tiene como objetivo generar información de línea base que sirva de punto de partida para futuras investigaciones.

\section{Metodología}

\section{1. Área de estudio}

El área de estudio seleccionada fue la microcuenca alta de la quebrada El Estero (MAQUE), ubicada al oeste de la ciudad de San Ramón, Costa Rica (figura 1). Se ubica entre las coordenadas $10^{\circ} 05^{\prime} 47^{\prime \prime}$ y $10^{\circ} \mathrm{O} 4^{\prime} 31^{\prime \prime}$, latitud norte y entre las coordenadas $84^{\circ} 28^{\prime} 20^{\prime \prime}$ y $84^{\circ} 28^{\prime} 41^{\prime \prime}$, longitud oeste, con una altitud promedio de $1078 \mathrm{msnm}$ (Mora, 2007). De acuerdo con el sistema de clasificación de Holdridge (1967), el área de estudio corresponde a la zona de vida de bosque premontano húmedo. En esta microcuenca quedan algunos humedales que son remanentes de lo que una vez fue un lago en el período Cuaternario (Bergoeing y Masís, 2013) y que, de acuerdo con Rodríguez-Arias y Silva (2017), fueron declarados por el Sistema Nacional de Áreas de Conservación de Costa Rica (SINAC) como una prioridad de conservación por su ubicación y representatividad.

\subsection{Descripción de los sitios de estudio}

Para propósitos de este trabajo, la zona de estudio se dividió en tres sitios: Bosque Municipal (BM), Bosque Demostrativo (BD) y Laguito (L), todos ubicados en la margen este de la quebrada El Estero (figura 1). En esta zona, la quebrada El Estero fluye de sur a norte por lo que el BM ocupa la parte más alta y el Laguito la más baja.

2.2.1. Bosque Municipal. El BM tiene un área aproximada de 7 ha y se caracteriza por ser bosque en regeneración y poseer gran cantidad de árboles de especies no nativas, como por ejemplo Casuarina, Eucalyptus y Cupresus (Guido y Rodríguez-Arias, 2009; Sibaja-Morales y Cartín, 2017), que fueron sembrados en la década de los 80 como parte de un proceso de reforestación (Matamoros y Badilla, 2017). Está rodeado por áreas de pasto para ganadería y por un cafetal. Junto al BM se observa un pequeño cauce que presumiblemente corresponde a una naciente, sin embargo, durante el período de estudio solamente se observó agua por escorrentía. El sitio cuenta con visitación no regulada debido a la falta de personal de control y vigilancia (Matamoros y Badilla, 2017).

2.2.2. Bosque Demostrativo. El BD tiene un tamaño aproximado de 5 ha y es en su mayoría bosque secundario con una pequeña área de tacotal (o,5 ha aproximadamente). El BD nació en la década de los 8 o como un intento de recrear la distribución de especies de árboles locales en remanentes de bosque de la región (Delgado, 1986), pero en la actualidad es el resultado de esa intervención y de la regeneración natural posterior. Su riqueza de especies 
arbóreas supera los 70 géneros (González y Barrantes, 2018). En su límite oeste, colinda con el cuerpo de agua de la quebrada El Estero y al este con el campus universitario Carlos Monge de la Universidad de Costa Rica (UCR). Por ser parte de la propiedad de la UCR y estar asociado a un proyecto de trabajo comunal (Araya y Almengor, 2017), recibe visitación controlada, pero también no controlada.

2.2.3. Laguito. Consta de un embalse artificial que cubre un área de aproximadamente 2 ha. Desde hace varios años no cuenta con un espejo de agua permanente, debido a la acumulación de sedimentos y la colonización de plantas (Chassoul y Rodríguez-Arias, 2018), por lo que la vegetación en este sitio está dominada por gramíneas como Leersia hexandra (Rodríguez-Arias y Silva, 2016). Este sitio está rodeado por terrenos urbanizados o en proceso de urbanización, por lo que probablemente también se dé la visitación no controlada en el sitio.

\subsection{Toma de datos}

La toma de datos inició en enero de 2016 y finalizó en octubre de 2017. Se usaron principalmente dos métodos para la recolección de los datos: transectos y cuadrantes de hojarasca. El primero consistió en recorrer senderos a lo interno de los diferentes sitios (BM, BD y L) siempre a una velocidad constante (aproximadamente $0,2 \mathrm{~km} / \mathrm{h}$ ) y haciendo búsqueda directa de los organismos de interés sobre el suelo y la vegetación que se encontraran a una distancia menor o igual a un metro, a cada lado del transecto. El segundo consistió en la ubicación al azar de varios cuadrantes de $100 \mathrm{~m}^{2}$, en los que se removió la hojarasca del suelo para recolectar cualquier anfibio o reptil que se hallara dentro. El esfuerzo de muestreo (ED) se contabilizó en horas persona (Hernández y Ramírez, 2013). Además, se registraron aquellas especies que fueron observadas por el autor en encuentros ocasionales, es decir, fuera de los eventos de muestreo, así como reportes de terceros siempre y cuando se tuvieran pruebas tangibles de su identificación (fotografías o capturas) y hubiesen ocurrido dentro del período de estudio (enero 2016-octubre 2017).

\subsection{Análisis de datos}

2.4.1. Riqueza de especies. Para estimar la riqueza de especies se tomaron en cuenta los datos obtenidos en los muestreos (transectos y cuadrantes), los encuentros ocasionales y los reportes hechos por terceros. Estos dos últimos se reportan en conjunto como registros ocasionales (Cuadro 1).

2.4.2. Índice de abundancia relativa. El índice de abundancia relativa (IAR), aquí reportado, se entiende como el número de capturas por evento de muestreo (cada evento tuvo el mismo esfuerzo de muestreo), multiplicado por un factor de 10. Para estimar dicho índice solamente se tomaron en cuenta los datos recopilados en los muestreos por transectos.
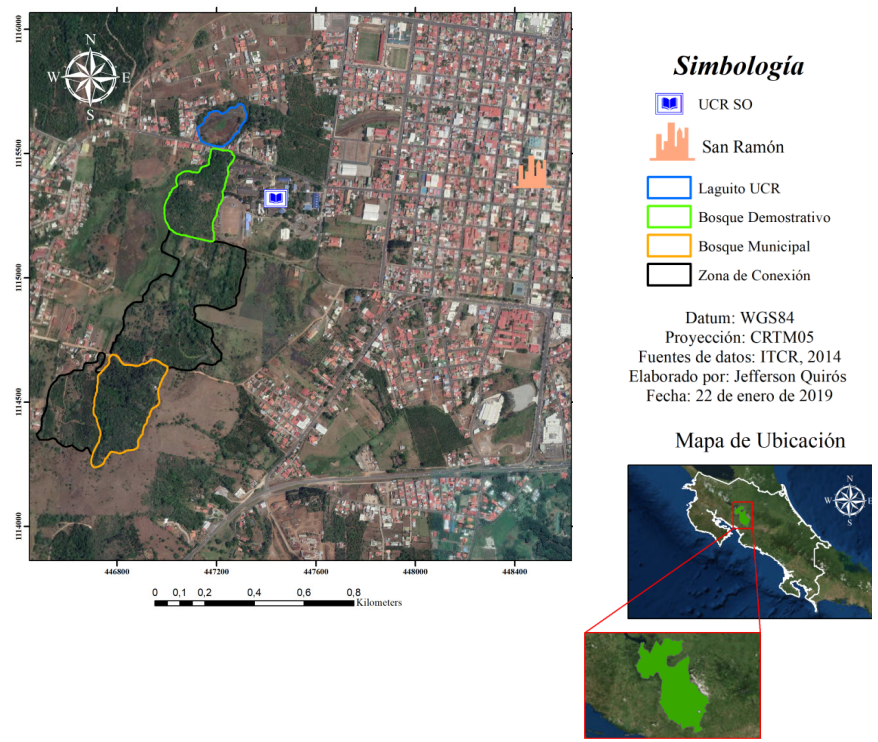

Figura 1. Área de estudio dentro de la microcuenca alta de la quebrada El Estero, San Ramón, Costa Rica. Nótese la composición urbana del paisaje. 


\section{Resultados}

Con un esfuerzo de muestreo de 120 horas persona, se registraron 19 especies representantes de 14 familias, 8 de reptiles y 6 de anfibios (Cuadro 1). La mayoría de las especies fueron registradas durante los muestreos (63 $\%, n=12$ ) pero una buena parte fue observada durante encuentros ocasionales ( $42 \%, \mathrm{n}=8$ ); solamente el sapo Incilius coccifer fue registrado tanto en los muestreos como de manera ocasional. De las 8 especies reportadas de manera ocasional (Cuadro 1), 7 corresponden a registros hechos dentro del campus universitario de la UCR y 1 , Enulius flavitorques, a un registro hecho en el Laguito.

De las especies encontradas, seis están totalmente asociadas al cuerpo de agua de la quebrada El Estero: las tortugas Kinosternon scorpioides, Chelydra acutirostris y Rhynoclemmys pulcherrima, así como las especies de ranas Rana forreri, Hyalinobatrachium fleischmanni y Dendropsophus microcephalus. Con respecto a los sitios de estudio, el BD fue el sitio con mayor riqueza de especies ( $n=9)$ y el BM el de menor ( $n=3$ ) (figura 2). En cuanto al índice de abundancia relativa de las especies, en ambos años de muestreo las más abundantes fueron Craugastor podiciferus $\left(\operatorname{IAR}_{2016}=10 \pm 11,9 ; \operatorname{IAR}_{2017}=5,5 \pm 11,3\right)$, H. fleischmanni ( $\left.\operatorname{IAR}_{2016}=13,7 \pm 19,9 ; \operatorname{IAR}_{2017}=4,4 \pm 8,8\right), y R$. forreri $\left(\mathrm{IAR}_{2016}=15,0 \pm 22,7 ; \mathrm{IAR}_{2017}=3,3 \pm 10,0\right)$ (figuras 3 y 4). El grupo de las serpientes fue el de mayor riqueza, con seis especies, aunque la mayoría fueron vistas en encuentros ocasionales y no así durante los muestreos.

\section{Cuadro 1.}

Lista de especies de anfibios y reptiles registradas durante el período 2016-2017 en la microcuenca alta de la quebrada El Estero, San Ramón, Costa Rica. M=menor preocupación; C= casi amenazado.

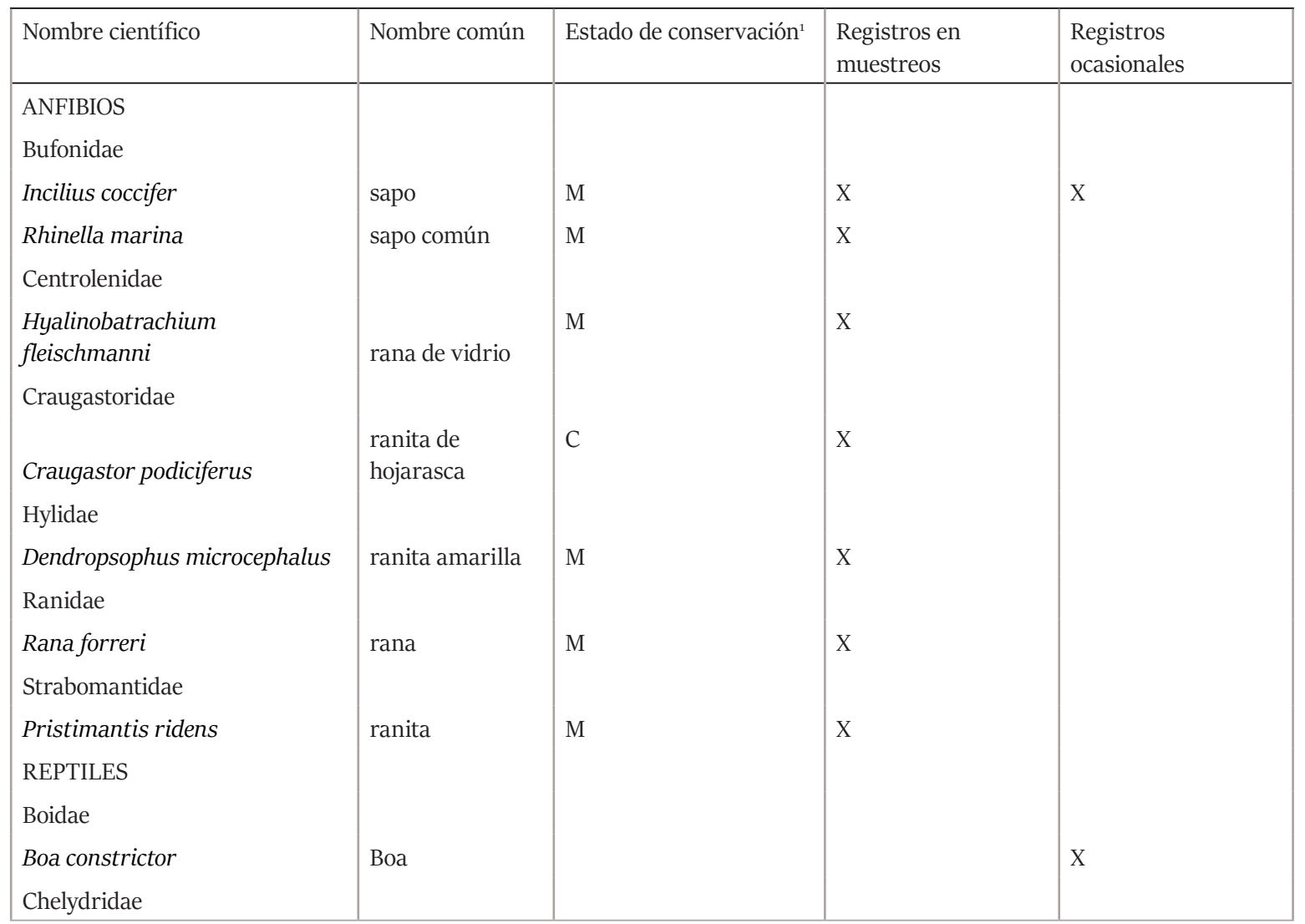




\begin{tabular}{|c|c|c|c|c|}
\hline Chelydra acutirostris & tortuga lagarto & & & $\mathrm{X}$ \\
\hline Colubridae & & & & \\
\hline Drymarchon melanurus & culebra & M & & $\mathrm{X}^{2}$ \\
\hline Lampropeltis triangulum & coral falsa & M & $\mathrm{X}$ & \\
\hline Mastigodryas melanolomus & culebra & M & & $\mathrm{X}$ \\
\hline Spilotes pulatus & mica & & & $\mathrm{X}^{2}$ \\
\hline Dactyloidae & & & & \\
\hline $\begin{array}{l}\text { Anolis cupreus } \\
\text { Dipsadidae }\end{array}$ & gallego & & $\mathrm{X}$ & \\
\hline Enulius flavitorques & culebra de tierra & M & & $\mathrm{X}^{2}$ \\
\hline $\begin{array}{l}\text { Geophis brachycephalus } \\
\text { Geomydidae }\end{array}$ & culebra de tierra & M & $\mathrm{X}$ & \\
\hline $\begin{array}{l}\text { Rhynoclemmys pulcherrima } \\
\text { Kinosternidae }\end{array}$ & tortuga pintada & M & & $X^{3}$ \\
\hline $\begin{array}{l}\text { Kinosternon scorpioides } \\
\text { Teiidae }\end{array}$ & tortuga candado & & $\mathrm{X}$ & \\
\hline Holocosus festivus & chisbala & M & $\mathrm{X}$ & \\
\hline
\end{tabular}

${ }^{1}$ Para aquellas especies que aparecen en la lista roja de especies de la Unión Internacional para la Conservación de la Naturaleza ${ }^{2}$ Fotografiada por terceros [IUCN] (IUCN, 2019).

${ }^{3}$ Capturada por terceros

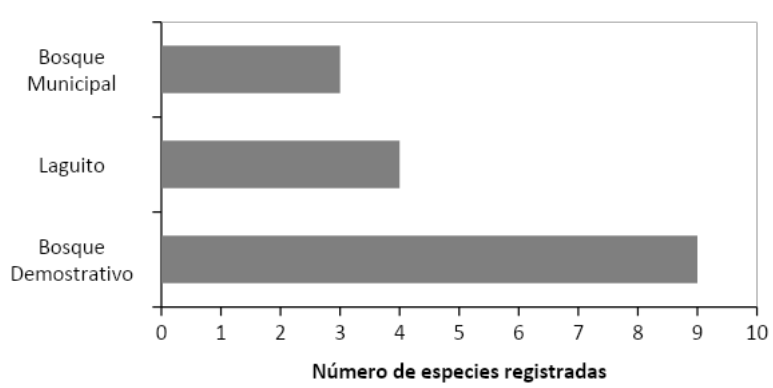

Figura 2.

Riqueza de especies según el sitio, en la microcuenca alta de la quebrada El Estero, 20162017.

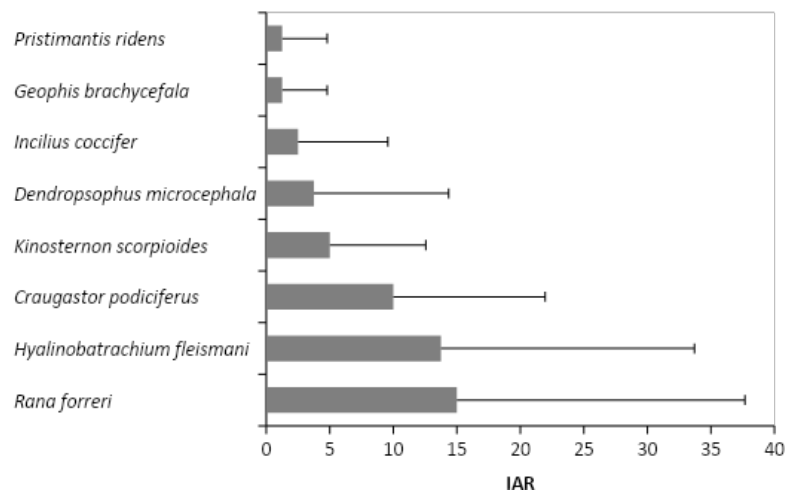

Figura 3.

Índice de abundancia relativa promedio (más desviación estándar) de las especies registradas en la microcuenca alta de la quebrada El Estero, durante el año 2016. 


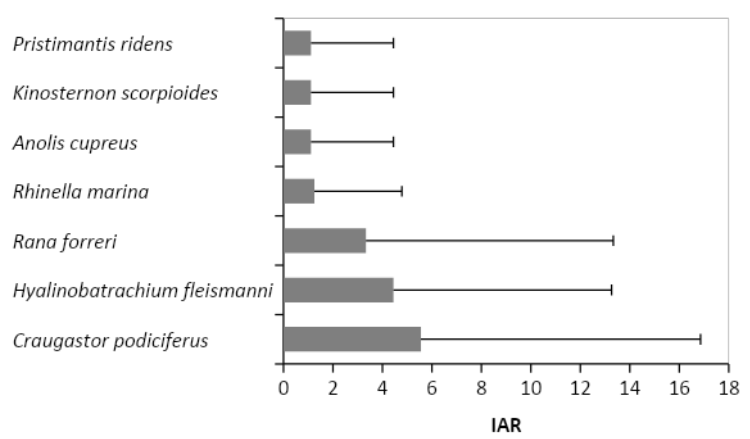

Figura 4. Índice de abundancia relativa promedio (más desviación estándar) de las especies registradas en la microcuenca alta de la quebrada El Estero, durante el año 2017.

\section{Discusión}

El número de familias de anfibios y reptiles representadas constituye casi el $30 \%$ de las familias que se pueden hallar en el territorio costarricense [47 según Bolaños et al. (2011)], lo cual es un buen número para un sitio tan pequeño e inmerso dentro del área urbanizada del cantón de San Ramón. En un trabajo similar, Abarca (2012) reportó 25 familias de anfibios y reptiles para la zona de El Rodeo, en el cantón de Mora, provincia de San José. No obstante, El Rodeo es un área de mucho mayor tamaño (2350 ha) que además posee los bosques primario y secundario distribuidos en tres zonas de vida: bosque premontano muy húmedo, bosque tropical húmedo y bosque tropical húmedo transición a premontano (Cascante-Marín, 2012; Ovares y Ramírez, 2012).

El número de especies registradas es menor al que se podría esperar si la zona mantuviera las condiciones previas a la modificación del hábitat causada por el ser humano. Por ejemplo, Morera y Sánchez (2016) reportaron 90 especies de anfibios y reptiles para la Reserva Biológica Alberto Manuel Brenes, un área protegida de bosque premontano, principalmente, y que podría ser un buen punto de cotejo gracias a su estado de conservación. Por su parte, Rovito et al. (2015) reportaron 30 especies en la Reserva Privada Nectandra, ubicada a unos $15 \mathrm{~km}$ de la ciudad de San Ramón. Probablemente la riqueza de especies en este último sitio sea mayor, pues el estudio de
Rovito y colaboradores (2015) fue de apenas una semana. No obstante, tanto Alberto Manuel Brenes (780o ha) como Nectandra (130 ha), son áreas de mayor tamaño que la cubierta en el presente estudio (apenas unas 15 ha).

La microcuenca alta de la quebrada El Estero se caracteriza por tener niveles variables de contaminación durante el año (Rodríguez-Arias y Silva, 2015). A pesar de ello, las especies de tortugas $C$. acutirostris, $K$. scorpioides y $R$. pulcherrima, parecen perdurar en estas condiciones. En el caso particular de $C$. acutirostris, ya se ha reportado su tolerancia a hábitats contaminados (e.g. Young-Valencia et al., 2018). En el presente trabajo, se encontró un juvenil de tortuga lagarto dentro de uno de los drenajes de la antigua planta de tratamiento de aguas residuales de la UCR (figura 5). Los individuos de las otras especies fueron hallados en el cuerpo de agua de la quebrada que, como ya se mencionó, presenta niveles variables de contaminación. Vale la pena mencionar que con respecto a $K$. scorpioides se tiene documentada su presencia en el sitio desde el año 1981, específicamente en el área del Laguito (Acuña et al., 1983), lo cual sugiere que la especie ha sido capaz de subsistir en un hábitat cada vez más degradado.

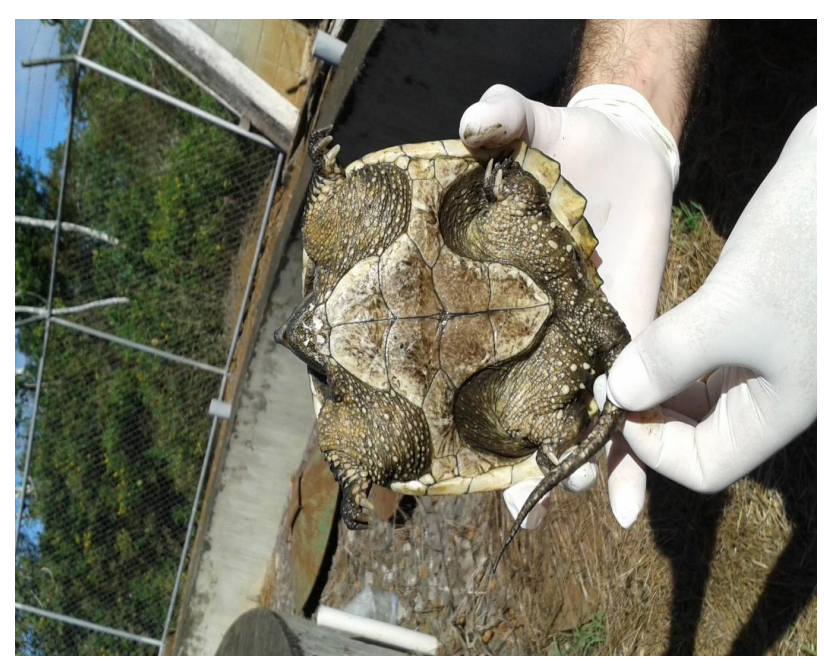

Figura 5. Ejemplar de tortuga lagarto (C. acutirostris), encontrada en los drenajes de la Planta de Tratamiento de Aguas Residuales de la UCR, Sede de Occidente, 23 de febrero de 2016. 
Por su parte, las ranas D. microcephalus y H. fleischmannni también son conocidas por tolerar cierto grado de contaminación (Bolaños et al., 2008; Buitrago-González y Vargas-Salinas, 2014; Coloma et al., 2010; Kubicki, 2007) y este también parece ser el caso de $R$. forreri en el presente trabajo. Estas especies fueron encontradas cerca o dentro del cuerpo de agua de la quebrada, contiguo al BD.

Con nueve especies registradas, el BD puede considerarse el principal refugio para las especies de anfibios y reptiles en la MAQUE. Varios factores podrían explicar esta situación. Por un lado, en el BD encontramos dos tipos de hábitat pues además del remanente de bosque se encuentra el cuerpo de agua de la quebrada que corre junto al mismo. Por otro lado, el pequeño parche de bosque posee una alta riqueza de especies forestales: González y Barrantes (2018) recientemente identificaron más de 900 especies de 72 géneros pertenecientes a 30 familias. Esto es importante porque dicho sitio es uno de los pocos remanentes de bosque premontano del Valle Central [sic] (González y Barrantes, 2018). En cambio el $\mathrm{BM}$ fue el sitio con la menor riqueza de anfibios y reptiles (apenas tres especies), pero se caracteriza por presentar una flora compuesta por muchos individuos de variedades arbóreas exóticas (Guido y Rodríguez-Arias, 2009; SibajaMorales y Cartín, 2017). Además, aunque junto al BM probablemente nacía una quebrada, la misma no tiene agua durante la mayor parte del año, incluso durante la estación lluviosa. Si las especies de árboles actuales fuesen paulatinamente sustituidos por ejemplares de flora autóctona, sería de esperar que en el futuro la riqueza de especies de los diferentes grupos de animales (incluida la herpetofauna) fuese mayor. Lo mismo se podría esperar si la quebrada tuviera agua de manera permanente y no solo por escorrentía.

Además de las ya mencionadas, hubo reportes de otras especies pero que no fueron registradas en este trabajo, debido a la ausencia de fotografías o de capturas. Por ejemplo, Micrurus nigrocinctus, Senticolis triaspis, Leptodeira spp, Imantodes spp, son algunas serpientes que con frecuencia son reportadas en la zona. A mediados del año 2017 se tuvo noticia de una serpiente terciopelo (Bothrops asper) a menos de $2 \mathrm{~km}$ del área. Lagartijas como Sceloporus malachiticus, Diploglossus bilobatus y
Gymnophtalmus speciosus también suelen ser observadas dentro del área de estudio (observación personal). Con respecto a anfibios, ranas como Smilisca phaeota y salamandras del género Oedipina aparecen con alguna regularidad (observación personal). Bolitoglossa striatula es otra salamandra que se encuentra en el área (Cartín, 2010). Por lo tanto, es de esperar que la riqueza de especies de la MAQUE sea mayor a la reportada, pese a lo intensivo del esfuerzo de muestreo realizado.

\section{Conclusiones}

Producto de este primer esfuerzo de línea base, se registraron 12 especies de reptiles (8 familias) y 7 de anfibios (6 familias) en la microcuenca alta de la quebrada El Estero. Seis de las 19 especies en total están asociadas a cuerpos de agua y se conocen por tolerar cierto grado de contaminación, razón por la que no sorprende su presencia en el área de estudio, que recibe aguas residuales de las urbanizaciones cercanas, sin ningún tipo de tratamiento previo. El Bosque Demostrativo de la Sede de Occidente de la Universidad de Costa Rica fue el sitio con la mayor riqueza de especies y esto viene a corroborar su importante papel como pequeño refugio para la vida silvestre que permanece en la periferia de la ciudad de San Ramón. La riqueza de especies dentro del área de estudio probablemente sea mayor, pues se conoce la presencia de otros organismos que no fueron registrados durante el período de estudio, por lo que sería recomendable realizar muestreos periódicos que permitan mantener un registro actualizado de la diversidad herpetofaunística del sitio.

\section{Agradecimientos}

A la Coordinación de Investigación de la Sede de Occidente y a la Vicerrectoría de Investigación de la Universidad de Costa Rica por el apoyo económico (Proyecto 540-B6-155). Al Sistema Nacional de Áreas de Conservación de Costa Rica (Resolución No013-2016-ACCVC-PI). A Karen Sibaja, Keilor Mora y María José Arias por el apoyo de campo. A Jefferson Quirós por la elaboración del mapa del área de estudio. A los evaluadores anónimos por sus oportunas observaciones y sugerencias. 


\section{Referencias bibliográficas}

Abarca, J.G. (2012). La herpetofauna de un bosque premontano: diversidad de anfibios y reptiles de El Rodeo. Brenesia, 77, 251-270.

Acuña, R.; Castaing, A. y F. Flores. (1983). Aspectos ecológicos de la distribución de las tortugas terrestres y semiacuáticas en el Valle Central de Costa Rica. Revista de Biología Tropical, 31(2), 181-192.

Araya, E. y M. Araúz-Almengor. (2017). Percepción pública de la existencia y utilidad del Bosque Demostrativo en San Ramón, Costa Rica. Cuadernos de Investigación, 8(2), 123-126.

Bergoeing, J. y R. Masís. (2013). Geomorfología del área de Palmares, San Ramón, Cerro Espíritu Santo, Costa Rica. Revista Geográfica, 154, 133-142.

Bolaños, F., Santos-Barrera, G., Solís, F., Ibáñez, R., Wilson, L.D., Savage, J., Lee, J., Trefaut Rodrigues, M., Caramaschi, U, Mijares, A. y J. Hardy. (2008). Dendropsophus microcephalus. The IUCN Red List of Threatened Species 2008: e.T55558A11318242. Recuperado el 15 de enero de 2018. http://dx.doi.org/10.2305/IUCN.UK. 2008. RLTS.T55558A11318242.en.

Bolaños, F., Savage, J. M y G. Chaves. (2011). Anfibios y Reptiles de Costa Rica. Listas Zoológicas Actualizadas UCR. Museo de Zoología UCR. San Pedro, Costa Rica. Recuperado el 15 de enero de 2018. http://museo.biologia.ucr.ac.cr/Listas/ LZAPublicaciones.htm.

Buitrago-González, W. y F. Vargas-Salinas. (2014). Dendropsophus microcephalus (Cope, 1886). Ranita amarilla de charca. Catálogo de Anfibios y Reptiles de Colombia, 2(2), 37-42.
Cartín, M. (2010). Bolitoglossa striatula: Predation. Herpetological Review, 41(2), 187.

Cascante-Marín, A. (2012). Ubicación, relieve y clima de la zona de El Rodeo. Brenesia, 77, 15-22.

Chassoul, M. y C. Rodríguez-Arias. (2018). Contaminación y disminución del embalse El Laguito, Costa Rica, por aguas urbanas: encuesta y propiedades físicoquímicas. Cuadernos de Investigación, 10(2), 409415 .

Coloma, L.A., Ron, S.R., Wild, E., Cisneros-Heredia, D., Solís, F., Ibáñez, R., Santos-Barrera, G. y B. Kubicki. (2010). Hyalinobatrachium fleischmanni. The IUCN Red List of Threatened Species 2010: e.T55014A11238651. Recuperado el 15 de enero de 2018. http://dx.doi.org/10.2305/IUCN.UK.2010-2. RLTS.T55014A11238651.en.

Delgado, S. (1986). Diseño de un bosque según patrones naturales de distribución en el Centro regional de Occidente-San Ramón [Tesis de Licenciatura]. Universidad de Costa Rica.

Domínguez-Vega, H. y I. Zuria. (2016). Ecología urbana y herpetofauna en México: potencial de un nicho poco explorado. Herreriana, 1, 31-34.

González, A. y T. Barrantes. (2018). Especies arbóreas identificadas en el Bosque Demostrativo de la Sede de Occidente, San Ramón, Alajuela, Costa Rica. Pensamiento Actual, 18(30), 78-124.

Gosá, A. y A. Arias. (2009). Estado de las poblaciones de anfibios en un parque urbano de Pamplona. Munibe, 57, 169-183.

Guido, I. y C. Rodríguez-Arias. (2009). Avifauna del bosque municipal José Figueres Ferrer, San Ramón, Alajuela, Costa Rica (Noviembre 2006 a Mayo 2009). Zeledonia, 13, 20-27. 
Hernández, U. y A. Ramírez. (2013). Distribución de la herpetofauna en cuatro tipos de vegetación del estado de Hidalgo, México. Estudios científicos en el estado de Hidalgo y zonas aledañas, 3, 5-12. Recuperado el 15 de abril de 2016. http:// digitalcommons.unl.edu/hidalgo/3.

Holdridge, L. R. (1967). Life zone ecology. Tropical Science Center.

Kubicki, B. (2007). Ranas de vidrio de Costa Rica. Instituto Nacional de Biodiversidad.

Matamoros, J. y A. Badilla. (2017). Estado Actual del Manejo Ambiental del Bosque Municipal José Figueres Ferrer, ubicado en el cantón de San Ramón de la provincia de Alajuela [Tesis de Licenciatura]. Universidad Estatal a Distancia.

Mora, C. (2007). Nueva propuesta de diseño para el embalse de regulación y uso múltiple "El Laguito", con el fin de prevenir las inundaciones en la ciudad de San Ramón [Tesis de Licenciatura]. Universidad de Costa Rica.

Morera, B. y R. Sánchez. (2016). Anfibios y reptiles de la Reserva Biológica Alberto Manuel Brenes. Pensamiento Actual, 15(25), 39-59.

Ovares, F. y M. Ramírez. (2012). El Bosque de El Rodeo: un legado para el país. Brenesia, 77, 1-14.

Piedra, L., Ramírez M. y L. Ovares. (2017). Las cuencas urbanas y su fauna: el caso del río Pirro, Heredia, Costa Rica. Biocenosis, 31(1-2), 58-61.

Rodríguez-Arias, C. y M. Silva. (2015). Calidad del agua en la microcuenca alta de la quebrada Estero en San Ramón de Alajuela, Costa Rica. Pensamiento Actual, 15(25), 85-97.

Rodríguez-Arias, C. y M. Silva. (2016). Vegetación acuática de los humedales de la microcuenca alta de la quebrada Estero, San Ramón de Alajuela, Costa Rica. Brenesia, 85-86, 9-20.
Rodríguez-Arias, C. y M. Silva. (2017). Los humedales de la quebrada Estero en San Ramón, Costa Rica: su importancia y estado actual. Revista Posgrado y Sociedad, 15(1), 13-26.

Rovito, S.; Devitt, T. y S. Devitt. (2015). First survey of the amphibians and reptiles of the Nectandra Cloud Forest Reserve, Alajuela, Costa Rica. Check List, 11(2), 1-8. DOI: https://doi.org/10.1556o/11.2.1570

Savage, J. (2002). The amphibians and reptiles of Costa Rica: A herpetofauna between two continents, between two seas. Chicago Press.

Scott N. y S. Limerick. (1983). Reptiles and amphibians. En: D. H. Janzen (Ed.), Costa Rican natural history (pp. 351-367). Chicago Press.

Sibaja-Morales, K. y M. Cartín. (2017). Unusual predation of Hoffmann's two-toed sloth (Choloepus hoffmanni) by a coyote (Canis latrans) in Costa Rica. Edentata 18, 88-91. DOI: 10.2305/IUCN. CH.2017.Edentata-18-1.3.en

Unión Internacional para la Conservación de la Naturaleza. (2019). Lista roja. https://www.iucnredlist.org/. Recuperado el 16 de diciembre de 2019.

Young-Valencia, K., Ortega-Guío, A. y A. Botero-Botero. (2018). Influencia de las variables de hábitat sobre la presencia de la tortuga pímpano (Chelydra acutirostris, Peters, 1862) (Chelydridae) en las quebradas Cajones y los Coclí, Quindio, Colombia. Revista colombiana de ciencia animal, 9(1), 6-13. 